\title{
Erfolgreiche Einführung von Enterprise Collaboration Systems: Work Design und Mitarbeiterpartizipation als Voraussetzung für Nutzungsakzeptanz?
}

\author{
Susanne Sczogiel ${ }^{1} \cdot$ Stephanie Schmitt-Rüth ${ }^{1}$ - Andreas Stöckl² • Olaf Struck²
}

Angenommen: 23. September 2021 / Online publiziert: 13. Oktober 2021

(c) Der/die Autor(en) 2021

\section{Zusammenfassung}

Enterprise Collaboration Systems (ECS) ermöglichen den digitalen Kontakt sowie Austausch von Informationen und Wissen zwischen Individuen, Teams und Unternehmen. Durch die Einführung von ECS am Arbeitsplatz verändern sich Arbeitsaufgaben, -abläufe, -anforderungen und Kommunikationswege. Die Ansprüche an die Gestaltung eines neuen Work Designs sowie der Mitarbeiterpartizipation steigen rasant. Die vorliegende Studie untersucht Arbeits- und organisationale Eigenschaften, welche mit der Nutzungsakzeptanz neuer Technologien zusammenhängen. Hierfür werden Work Design (in Form von Job-Stressoren und -Ressourcen), Vorerfahrungen mit Informationstechnologien und Mitarbeiterpartizipation untersucht. In einer schriftlichen Befragung $(N=212)$ in zwei Unternehmen, in denen ECS eingeführt wird, wurden Mitarbeitende bzgl. der Eigenschaften ihrer Arbeit, ihrer Organisation und ihrer eigenen Einstellungen bzgl. Technologien befragt. Es zeigten sich Zusammenhänge zwischen einigen Job-Stressoren und -Ressourcen und der Nutzungsakzeptanz. Auch die Vorerfahrungen mit Technologien hing mit Nutzungsakzeptanz zusammen, während Mitarbeiterpartizipation keinen Zusammenhang aufwies.

Praktische Relevanz: Die Studie identifiziert potenzielle Arbeits- und organisationale Eigenschaften, welche vom Arbeitgeber beeinflussbar sind, die mit der Nutzungsakzeptanz von neuen Kommunikations- und Wissenstransfertechnologien, im Speziellen mit ECS, zusammenhängen. Die Erkenntnisse können bei der Einführung solcher neuen Technologien am Arbeitsplatz zur Verbesserung der Nutzungsakzeptanz verwendet werden.

Schlüsselwörter Arbeitsgestaltung $\cdot$ Work Design $\cdot$ Nutzungsakzeptanz $\cdot$ Digitalisierung $\cdot$ Mitarbeiterpartizipation

Susanne Sczogiel, M. Sc.

susanne.sczogiel@iis.fraunhofer.de

1 Fraunhofer-Institut für Integrierte Schaltungen IIS, Nordostpark 84, 90411 Nürnberg, Deutschland
2 Professur für Arbeitswissenschaft, Otto-Friedrich-Universität, Feldkirchenstraße 21, 96052 Bamberg, Deutschland 


\title{
Successful implementation of enterprise collaboration systems: work design and employee participation as conditions for user acceptance?
}

\begin{abstract}
Virtual communication as well as virtual teams are becoming more and more vital for companies, as shown by the pandemic. In order to manage the transition into the digital world of communication and knowledge sharing, companies start using technologies such as Enterprise Collaboration Systems (ECS), which need to be accepted by the employees in order to be successfully used. How can employers facilitate user acceptance? This study sets out to find work and organizational characteristics that relate to user acceptance. For this aim, the authors examined work design (operationalized as job stressors and job resources), experience with technologies and employee participation. With the help of a survey in two companies, which are currently introducing ECS, employees $(N=212)$ reported about work and organizational characteristics as well as their own attitudes towards technology. Results show relations between certain job stressors, job resources and user acceptance. Experiences with technology also related to user acceptance. Employee participation and user acceptance didn't show any relations.

Practical Relevance: This study identifies potential work and organizational characteristics open to influence by the employer, which are related to user acceptance towards new communication and knowledge sharing technologies, in this case Enterprise Collaboration Systems. The findings can potentially be used for the implementation of such technologies at the workplace in order to facilitate higher user acceptance.
\end{abstract}

Keywords Work design $\cdot$ Job design $\cdot$ User acceptance $\cdot$ Digitalization $\cdot$ Employee participation

\section{Einleitung}

Die Digitalisierung der Arbeitswelt verändert nicht nur Technik, Produkte und Geschäftsmodelle, sondern ist mit einer außerordentlichen Flexibilisierung von Arbeitszeiten, Arbeitsorten, Arbeitsinhalten, Arbeitsanforderungen und Kommunikation verbunden. Nicht zuletzt ist es die Pandemie, die dies unter Beweis stellt: Mehr und mehr sind Mitarbeitende am (flexiblen) Arbeitsplatz abhängig von digitalen Kommunikationsmedien. Um die beschleunigten und dezentralisierten Kommunikations- und Kooperationsprozesse zu meistern, bedarf es IT-gestützter Kooperationssysteme. Diese finden immer mehr Einzug nicht nur in Großkonzerne, sondern auch in kleinere und mittelständische Unternehmen (KMUs): Sogenannte Enterprise Collaboration Systems (ECS; Schubert und Williams 2013) wie Microsoft Teams, JIRA und ähnliche Softwares bzw. Technologien werden in den Bereichen Kommunikation, Projektorganisation und Wissensmanagement eingesetzt (Koch und Richter 2009). Infolge dezentraler und flexibler Kommunikation, einem vereinfachten Teilen von Wissen und der Möglichkeit der digitalen Abbildung ganzer Organisationsstrukturen heben ECS digitales kollaboratives Arbeiten auf eine neue Ebene. Vor allem die genannten KMUs müssen hierfür häufig neue Strukturen und Prozessabläufe aufbauen. Diese Schritte in Richtung einer flexiblen und digitalen Kommunikation werden in sehr vielen Unternehmen als eine wichtige jedoch nicht einfach zu bewältigende Herausforderung angesehen.

Die vorliegende Studie entstand im Rahmen der Forschung über die Einführung von Enterprise Collaboration
Systems in Unternehmen ${ }^{1}$. Untersucht werden unter anderem die Auswirkungen auf Akzeptanz und Belastungserleben sowie die Veränderung von Kommunikation und das Teilen von Wissen und Informationen innerhalb der Belegschaft. Ein besonderer Fokus liegt hierbei auf den Faktoren, die die Akzeptanz der Belegschaft für das neue ECS begünstigen. Um Ergebnisse zu erzielen, die weiteren KMUs bei der Einführung von ECS helfen können, stellen wir uns die Frage, welche Faktoren in der Arbeitssituation von Mitarbeitenden existieren, die die Nutzungsakzeptanz verbessern. Dabei konzentrieren wir uns vor allem auf individuelle sowie organisationale Faktoren, welche von Entscheidern im Unternehmen wie Führungskräften beeinflusst werden können: Aspekte der Arbeitsgestaltung (Work Design), Partizipationsmöglichkeiten für Mitarbeitende sowie die Erfahrungen im Umgang mit Technik am Arbeitsplatz.

\section{Theorien}

\subsection{Nutzungsakzeptanz und Widerstand}

Um zu erklären, warum neue Technik scheitert, hat sich das Feld der Akzeptanzforschung etabliert. Technikakzeptanzforschung verfolgt das Ziel, Modelle und Theorien auf-

\footnotetext{
${ }^{1}$ Forschungsprojekt „KoMiK - Digitale Kooperationssysteme im Mittelstand: Innovative Kommunikations- und Kooperationsprozesse in der digitalen Arbeitswelt“ (gefördert vom Bundesministerium für Bildung und Forschung und dem Europäischen Sozialfonds; Laufzeit 2019-2022).
} 
zustellen, um zu erklären und vorherzusagen, wann neue Technik von Individuen angenommen wird und welche Faktoren die Entstehung von Akzeptanz fördern oder hemmen. Kollmann (1999) betont dabei, dass der individuelle Akzeptanzprozess stets mehrstufig abläuft: von einer affektiven und kognitiven Einstellungsbildung (attitude) über die Bildung einer Verhaltensabsicht (behavioral intention) bis hin zum tatsächlichen Verhalten (actual behavior). Im organisationalen Kontext bezieht sich Verhalten speziell auf die Nutzung einer neuen Technik am Arbeitsplatz (intention to use/actual use). 1989 stellten Davis, Bagozzi und Warshaw. das erste Technologieakzeptanzmodell (Technology Acceptance Model, TAM) seiner Art vor, um Nutzungsakzeptanz mit dem Fokus auf Informationstechnologien zu modellieren. Dabei wurde der Verhaltensintention als Prädiktor für das tatsächliche Verhalten eine entscheidende Bedeutung beigemessen. Das TAM gilt bis heute als eines der am häufigsten verwendeten Akzeptanzmodelle. Es wurde im Laufe der Zeit um eine Vielzahl an potenziellen Einflussfaktoren auf die Nutzungsakzeptanz ergänzt. Mit ihrer Unified Theory of Acceptance and Use of Technology (UTAUT) verfolgten Venkatesh et al. (2003) das Ziel, die breite Theoriebasis der Nutzungsakzeptanz bezogen auf neue Technik - unter Einbezug von acht prominenten Akzeptanzmodellen - allgemeingültig zusammenzuführen. Die Autoren testeten das UTAUT-Modell mithilfe einer Längsschnittstudie bei Angestellten in vier Organisationen, die mit einer neuen Technologie am Arbeitsplatz arbeiten sollten. Als abhängige Variable wurde Nutzungsakzeptanz sowohl in Form von Verhaltensabsicht als auch in Form tatsächlicher Nutzung untersucht.

Grundsätzlich zeigt sich, dass die Nutzung neuer Technologien in Unternehmen stets mit Veränderung verbunden ist: sowohl bezogen auf organisationale Aspekte als auch auf potenzielles Nutzungsverhalten. Für das Scheitern von Innovationen und Change Prozessen wird Widerstand gegenüber Veränderung als einer der Hauptfaktoren angenommen (Lauer 2010). Widerstand kann sowohl als eine kognitive, einstellungsbasierte als auch verhaltensbasierte Dimension konzeptualisiert werden, stellt jedoch in allen Varianten das Ergebnis einer negativen Bewertung eines Objektes dar (Heidenreich 2012). Oreg (2003) operationalisiert die Neigung einer Person, sich Veränderungen zu widersetzen oder zu vermeiden, Veränderungen generell abzuwerten und Veränderungen in verschiedenen Kontexten und bei verschiedenen Arten von Veränderungen als unangenehm zu empfinden (ebd. S. 680) als eine TraitVariable: Widerstand gegen Veränderung. Auf Basis dieser grundlegenden Persönlichkeitseigenschaft kann erklärt werden, warum manche Menschen eher dazu neigen als andere, neue Technologien, anzunehmen, und damit ebenfalls Rückschlüsse auf die Nutzungsakzeptanz ermöglichen. Um unsere Forschungsfrage zu beantworten, welche Faktoren in der Arbeitssituation einer Mitarbeiterin und eines Mitarbeiters existieren, die die Nutzungsakzeptanz verbessern, konzeptualisieren wir Nutzungsakzeptanz gemäß des UTAUT durch die Verhaltensintention, die neue Technologie (hier: ECS) zu nutzen, sowie mit Hilfe des Widerstands gegenüber Veränderungen. Auf der Suche nach solchen Faktoren untersuchen wir auch Work Design.

\subsection{Work Design}

Ein wichtiger Aspekt der Arbeit, der von der Organisation sowie von Führungskräften beeinflusst werden kann, ist die Gestaltung der Arbeit. Parker (2014) definierte den Begriff Work Design wie folgt: „Der Inhalt und die Organisation der Arbeitsaufgaben, Aktivitäten, Beziehungen und Verantwortlichkeiten“ im Arbeitskontext. In der langen Forschungstradition der Arbeits- und Organisationspsychologie können fünf Cluster der Work Design-Forschung identifiziert werden (Parker et al. 2017): Soziotechnische Systeme und autonome Arbeitsgruppen, das Job-Characteristics-Modell (JCM), das Job-Demands-Control-Modell (JDCM), das Job-Demands-Resources-Modell (JDRM) sowie die Rollentheorie. Vor allem das zunächst in der Burnout-Forschung konzipierte JDRM hat in der Forschung über den Einfluss von Arbeit auf das Befinden von Arbeitnehmerinnen und Arbeitnehmern insgesamt eine große Bedeutung erlangt (Demerouti et al. 2001). Das JDRM kann als Erweiterung des JDCM gesehen werden. Das JDCM (Karasek 1979, 1998) betrachtet Stressoren in Form von job demands (vor allem zu viel Arbeitslast und Zeitdruck) als Auslöser für Stress und gesundheitliche Schäden. Das Modell postuliert, dass viel Handlungsspielraum, das heißt Autonomie und Freiheiten in der Bearbeitung der Arbeitsaufgaben und der Gestaltung des Arbeitsalltags, den Zusammenhang zwischen hohen stressbehafteten Arbeitsanforderungen und den schädlichen gesundheitlichen Folgen positiv beeinflussen kann. Obwohl es hierfür reichlich Belege gibt, ist unklar, warum nur Handlungsspielraum in dieses Modell aufgenommen wurde und weitere Ressourcen, die vermutlich den Zusammenhang zwischen Anforderung und Stress ebenfalls abfedern könnten, vernachlässigt wurden. Zum einen wurde das JDCM selbst um die Ressource der sozialen Unterstützung erweitert (Johnson und Hall 1988). Zum anderen wurden in der Forschung mehr und mehr Ressourcen beobachtet, die einen puffernden Effekt zwischen stressbehafteten Arbeitsanforderungen (wie Arbeitslast oder die hohe Komplexität einer Aufgabe) und schädlichen gesundheitlichen Folgen ausüben. Demerouti und Kolleg/innen (2001) systematisierten diese Erkenntnisse. Ihr $J D R M$ (Abb. 1) vereint alle Ressourcen, die dem Work Design-Begriff entsprechen oder Jobcharakteristika sind (siehe Abb. 1). Gemäß des JDCM wird in dem Modell angenommen, dass alle Ressourcen, nicht nur der Handlungsspiel- 
Abb. 1 Das Job-Demands-Ressources-Modell. (Bakker und Demerouti 2007)

Fig. 1 The job demands resources model. (Bakker und Demerouti 2007)

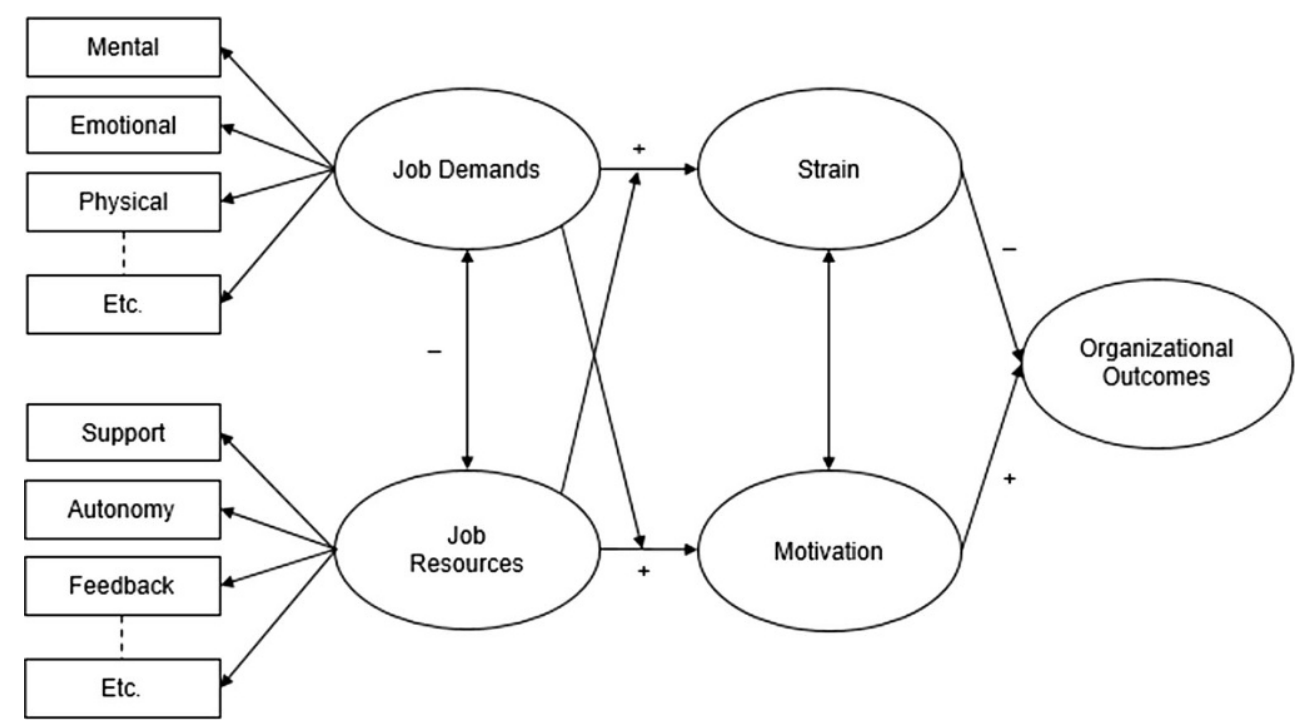

raum, den Zusammenhang zwischen Anforderungen und dem schädlichen Strain auf die Mitarbeitenden abpuffern. Das Modell hat sich über die Zeit zum State of the art in der Stressforschung der Arbeits- und Organisationspsychologie etabliert: Über einen Zeitraum von 15 Jahren wurde der erste Entwurf dieses Modells sowohl empirisch bestätigt als auch durch weitere theoretische Überlegungen und Zusätze weiterentwickelt (Bakker und Demerouti 2017).

Die Stressoren und Ressourcen, die in Untersuchungen über das JDRM einen Einfluss auf das Befinden von Mitarbeiterinnen und Mitarbeitern genauer betrachtet werden, sind unter anderem auch job characteristics, die im JCM (Hackman und Oldham 1975, 1976; Hackman 1980) zu finden sind. In ihrer Entwicklung des Job Diagnostic Surveys (1975) fanden Hackman und Oldham vor allem fünf Eigenschaften von Arbeitsaufgaben, die Einfluss auf verschiedene Outcomes bei den Mitarbeitenden haben wie auf intrinsische Motivation, Arbeitszufriedenheit oder die Qualität der Arbeitsleistung. Diese fünf job characteristics sind Anforderungsvielfalt (Kann eine Person ihr ganzes Skillset bei der Erledigung einer Aufgabe einsetzen?), Ganzheitlichkeit der Aufgabe (Kann eine Person ihre Arbeitsaufgabe von Anfang bis Ende ganzheitlich durchführen?), Wichtigkeit der Aufgabe (Sieht die Person ihre Arbeitsaufgabe für die Organisation als bedeutsam an?), Autonomie (Wie viel Handlungsspielraum hat eine Person bei der Erledigung einer Arbeitsaufgabe?) und Rückmeldung durch die Tätigkeit (Kann eine Person bei Vollendung einer Arbeitsaufgabe durch die Aufgabe selbst Feedback erfahren?). Hier zeigt sich bereits, dass solche Kernelemente des Work Designs wie job characteristics Zusammenhänge mit verschiedenen Outcomes haben; im JCM auf Motivation und Leistung (Hackman und Lawler 1971; Hackman 1980), im JDCM und JDRM auf Stresserleben und positive Befindensoutcomes wie Engagement (Bakker und Demerouti 2017).
Sowohl das JDCM als auch das JDRM behelfen sich der Rollentheorie, indem sie Rollenkonflikt sowie Rollenambiguität, das heißt, die Wahrnehmung widersprüchlicher Erwartungen an ihre Rolle, als Stressoren miteinbeziehen. Vor der Konzeption des JDCMs zeigten schon Levinson und Kollegen (1965) den Zusammenhang zwischen Rollenkonflikt bzw. Rollenambiguität und Stress am Arbeitsplatz auf, welcher in weiteren empirischen Studien repliziert werden konnte (Mérida-López et al. 2017; Olivares-Faúndez et al. 2014; Schwab und Iwanicki 1982).

\subsection{Work Design und Nutzungsakzeptanz}

In der von Parker et al. (2017) identifizierten Work DesignPerspektive der soziotechnischen Systeme geht es vor allem um die Bildung von autonomen Arbeitsgruppen als Antwort darauf, dass die Veränderung der Arbeit durch fortgeschrittene Technik von Arbeitnehmenden mehr Selbstmanagement forderte. Diese Richtung der Work Design-Forschung wurde jedoch maßgeblich von Studien im Bereich der Kohleförderung oder des verarbeitenden Gewerbes der 50er bis 70er-Jahre initiiert (Parker et al. 2017). Technik heute ist vernetzter. Fortgeschrittene Mensch-Technik-Interaktionen sind von Technik geprägt, die sich immer schneller verändern und immer tiefer den ganzen Arbeitsablauf durchdringen. Mittels Digitalisierung wird der gesamte Arbeitsplatz neugestaltet. Arbeitsaufgaben, -mittel und -schritte werden durch die digitale Transformation verändert oder sogar ersetzt; das beginnt bei der Umstellung von Papier auf digitale Dokumente, der elektronischen Zeiterfassung, dem Einführen neuer Computersysteme und vielen weiteren vermeintlichen „Kleinigkeiten“, und endet bei der Zusammenarbeit mit künstlicher Intelligenz und selbstlernenden Algorithmen, mit Robotern am Arbeitsplatz und Software, die ganze Arbeitsschritte selbst übernehmen. Die verschie- 
Abb. 2 Hypothesen 1 und 2. (Eigene Abbildung)

Fig. 2 Hypotheses 1 and 2. (Own figure)

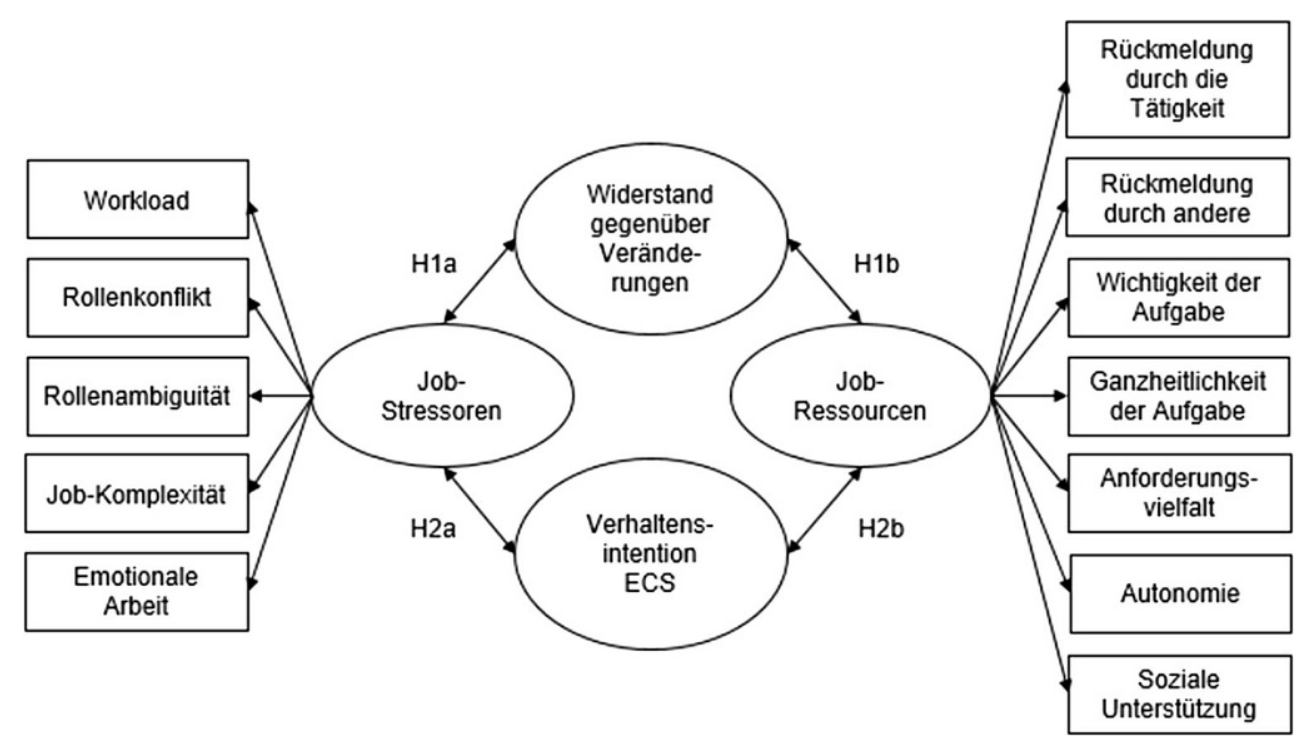

denen Dimensionen der Work Design-Forschung arbeiten vor allem mit arbeits- und organisationspsychologisch relevanten Outcomes wie Arbeitszufriedenheit, Motivation oder Stresserleben. Erste Hinweise, dass solche Outcomes auch mit Nutzungsakzeptanz einhergehen, gibt es schon: Schraeder, Swamidass und Morrison (2006) fanden beispielsweise in einer Studie im Gesundheitssektor heraus, dass Mitarbeitende, die hohe Rollenambiguität verspüren, negativer auf Technologieveränderungen im Arbeitskontext reagieren. In dieser Studie wurden auch zwischen Job-Stress und Einstellung gegenüber Veränderungen bzw. Technologieakzeptanz Zusammenhänge gefunden. Solche Zusammenhänge zwischen dem Wohlbefinden der Mitarbeitenden und der Einstellung gegenüber Veränderungen bzw. Nutzungsakzeptanz lässt sich auch in einer Studie mit koreanischen Expats in den USA (Lee et al. 2009) beobachten.

$\mathrm{Um} \mathrm{zu}$ verstehen, welche Faktoren in der Arbeitssituation einer Mitarbeiterin und eines Mitarbeiters existieren, die die Nutzungsakzeptanz verbessern, fragen wir daher zunächst, ob Personen, die einen Widerstand gegenüber Veränderung zeigen auch eher angeben, dass sie mehr Stressoren und weniger Ressourcen in ihrer Arbeitsgestaltung wahrnehmen. Somit wäre ein erster Schritt getan, um danach erforschen zu können, welche Kausalität diese Beziehung hat, und inwieweit man hier an der Person oder am Work Design ansetzen kann.

Als weitere Einflussgröße von Nutzungsakzeptanz wird die Verhaltensintention, eine neue Technologie zu nutzen, untersucht: Wir stellen uns die Frage, ob Work Design, operationalisiert durch Jobcharakteristika, die sich in Stressoren und Ressourcen aufteilen, mit der Absicht, eine neue Technologie für das Bearbeiten von Arbeitsaufgaben zu nutzen, zusammenhängt.
Das Untersuchungsmodell der vorliegenden Studie zur Technikakzeptanz und Work Design (Hypothesen 1 und 2) lässt sich Abb. 2 entnehmen.

Deswegen formulieren wir $\mathrm{zu}$ den Zusammenhängen zwischen Job-Stressoren, Job-Ressourcen und Widerstand gegenüber Veränderungen bzw. die Verhaltensabsicht, das neue ECS zu nutzen, folgende Hypothesen:

Hypothese 1a: Job-Stressoren und Widerstand gegenüber Veränderungen hängen positiv zusammen.

Hypothese 1b: Job-Ressourcen und Widerstand gegenüber Veränderungen hängen negativ zusammen.

Hypothese 2a: Job-Stressoren und die Verhaltensabsicht, ECS zu nutzen, hängen positiv zusammen.

Hypothese 2b: Job-Ressourcen und die Verhaltensabsicht, ECS zu nutzen, hängen negativ zusammen.

Dadurch, dass wir explorieren wollen, ob Job-Stressoren und Job-Ressourcen ebenfalls als trennbar negative und positive Einflussfaktoren auf die Technikakzeptanz von ECS wirken, formulieren wir die Hypothesen zunächst ungerichtet. Bei der Untersuchung handelt es sich nicht um eine Modellerweiterung, sondern um die Überprüfung, ob sich die im JDRM enthaltenen Job-Stressoren und Job-Ressourcen zu Technikakzeptanz ähnlich verhalten wie zu Befindensoutcomes.

Um der Beziehungsebene der Definition von Work Design (Parker 2014) gerecht zu werden, werden in der vorliegenden Arbeit ergänzend Beziehungskonstrukte sowohl in den Stressoren als auch in den Ressourcen verwendet. In den Stressoren wird auch die emotionale Arbeit untersucht, die schon in zahlreichen Burnout-Studien als Stressor identifiziert wurde (Jeung et al. 2017; Zapf 2002). Als Beziehungsressource wird, neben der sozialen Unterstützung, die Rückmeldung durch andere (Feedback) herangezogen. Auch Feedback konnte in vielen Studien als Ressource mit 
Tab. 1 Untersuchte Stressoren und Ressourcen

Table 1 Examined stressors and resources

\begin{tabular}{ll}
\hline Stressoren & Ressourcen \\
\hline Workload & Rückmeldung durch die Tätigkeit \\
Rollenkonflikt & Rückmeldung durch andere \\
Rollenambiguität & Wichtigkeit der Aufgabe \\
Job-Komplexität & Ganzheitlichkeit der Aufgabe \\
Emotionale Arbeit & Anforderungsvielfalt \\
& Autonomie \\
& Soziale Unterstützung \\
\hline
\end{tabular}

positiven Outcomes bestätigt werden (Battistelli et al. 2013; Katsikea et al. 2011). In Tab. 1 kann entnommen werden, welche Stressoren und Ressourcen die vorliegende Studie aus der Tradition der Work Design-Forschung zur Untersuchung der Hypothesen heranzieht.

Neben Work Design gibt es weitere Merkmale, welche von der Arbeitsorganisation beeinflusst werden können und die Einfluss auf das Erleben von Mitarbeitenden am Arbeitsplatz haben, die nicht den Inhalt und die Organisation der Arbeitsaufgaben, Aktivitäten, Beziehungen und Verantwortlichkeiten (Parker 2014) betreffen, die jedoch einen Zusammenhang mit Technikakzeptanz haben könnten. Die zwei Merkmale, die wir hierbei zusätzlich betrachten, sind die Vorerfahrung mit Informationstechnologien und die Möglichkeit der Partizipation im Unternehmen.

\subsection{Vorerfahrung mit Informationstechnologien}

Positive Erfahrungen durch die Arbeit mit Techniken steigern die technische Selbstwirksamkeit (Schreder et al. 2013) und sie können durch die Organisation gezielt aufgebaut oder gefördert werden.

Erste Ansätze zur Erfassung von positiven Vorerfahrungen finden sich bereits in der zweiten Version des vielfach verwendeten Technology Acceptance Model (TAM2), in welche Erfahrung (Experience) als Moderator aufgenommen wurde (Venkatesh und Davis 2000). Auch im UTAUTModell und in der dritten Version des TAM (TAM3) wurde der positive Effekt von Vorerfahrungen mit einem spezifischen Programm auf die Nutzungsabsicht dieses Programms nachgewiesen (Venkatesh und Davis 2000; Venkatesh und Bala 2008). Es wurde in den betrachteten Studien die Erfahrung mit dem eingeführten System selbst gemessen. Im Zuge von innerbetrieblichen Modernisierungen und der Einführung einer neuen Technik bestehen i.d.R. keine konkreten Erfahrungen mit dem neuen System. Gleichwohl verfügen die Mitarbeitenden häufig über allgemeinere Vorkenntnisse. In den von uns untersuchten Unternehmen bestehen keine Erfahrungen mit ECS, aber Erfahrungen mit Datenbanken, welche als Vorgängertechnologie zu ECS angesehen werden können. Wir vermuten, dass ein empfunde- ner positiver Einfluss der Arbeit mit digitalen Datenbanken auf die Produktivität hilft, Vorbehalte und Unsicherheiten im Umgang mit Technik abzubauen und deren konstruktives Potenzial auf die eigene Arbeit zu erfassen (Compeau \& Higgins 1995; Schreder et al., 2013). Anders als in bisherigen Studien (Venkatesh und Davis 2000; Venkatesh und Bala 2008) wollen wir uns jedoch explizit auf den Einfluss bereits implementierter Vorgängertechniken auf die Nutzungsabsicht von Folgetechniken konzentrieren. Ein neues System wird, bei positiver Vorerfahrung mit Vorgängertechniken, mit weniger Skepsis bedacht und eher zur Unterstützung der eigenen Arbeit verwendet. Wir formulieren daher:

Hypothese 3: Positive Erfahrungen mit Datenbanken als Vorgängertechnologie stehen in positivem Zusammenhang zur Nutzungsabsicht von ECS.

\subsection{Partizipation und Gerechtigkeit}

Die bereits erläuterten Faktoren beziehen sich auf die Einstellung einzelner Personen und der Struktur ihrer Arbeitsplätze zur Nutzungsabsicht von ECS. Weniger beachtet wurde bisher die Frage nach dem Einfluss organisationaler Faktoren, welche gerade im Einführungsprozess einer Technik eine große Rolle spielen können (Cooper und Zmund 1990; Venkatesh und Bala 2008). Eine besondere Eigenschaft der dieser Studie zugrundeliegenden Stichprobe ist das betriebliche Setting. Studien zum Thema der Technikakzeptanz erforschten die Nutzung neuer IKTSysteme unter Personen, wenn diese auf freiwilliger Basis genutzt werden können (Venkatesh und Davis 2000; Venkatesh und Bala 2008). In einem betrieblichen Setting ist es jedoch eher der Regelfall, dass die Nutzung einer neuen Technik nicht optional, sondern vorgeschrieben ist. Relevant für den Betrieb ist der Moment der Entscheidung zur Einführung.

Bei der Ausgestaltung des Entscheidungsprozesses wird angenommen, dass Unternehmensentscheidungen von Mitarbeitenden eher akzeptiert werden, wenn sie die Verfahren, die zur Entscheidung geführt haben, als gerecht empfinden (Leventhal 1980). Mögliche Widerstände unter den Mitarbeitenden, welche sich unter anderem aus einem als ungerecht empfundenen Entscheidungsprozess ergeben, könnten dann eine ineffiziente Nutzung der Technik nach sich ziehen (Cooper und Zmud 1990; Wong et al. 2018).

Die Gerechtigkeit eines Entscheidungsverfahrens hängt nach Leventhal (1980) von mehreren Regeln ab. Einheitliche Regeln für alle Beteiligten (Consistency Rule), Berücksichtigung der Interessen und Werte aller beteiligten Personen (Representativeness Rule), Verhinderung der Durchsetzung von Eigeninteressen aller am Prozess Beteiligten (Bias-Suppression Rule), Orientierung an geltenden ethischen und moralischen Standards (Ethicality Rule), Transparenz bezüglich der zur Meinungsbildung relevanten Informatio- 
nen (Accuracy Rule) und Widerspruchsmöglichkeiten (Correctability Rule).

Insbesondere aus der Correctability und der Representativeness Rule wird die Notwendigkeit der aktiven Partizipation der Mitarbeitenden an Entscheidungsprozessen erkennbar, um ein Empfinden von Verfahrensgerechtigkeit sicher zu stellen. Die Möglichkeit mitzubestimmen, erhöht die Wahrscheinlichkeit, dass der Entscheidungsprozess durch die Mitarbeitenden als gerecht empfunden wird, womit der Beschluss und die daraus resultierenden Folgen von Mitarbeitenden eher akzeptiert werden.

Hypothese 4a: Eine hohe Partizipation der Mitarbeitenden ist mit höherer Nutzungsabsicht des ECS durch die Mitarbeitenden verbunden.

Hypothese 4b: Eine hohe Partizipation der Mitarbeitenden ist mit niedrigerem Widerstand gegen Veränderungen verbunden.

In dem in dieser Studie zugrundeliegenden Fragebogen wurde die Partizipation der Mitarbeitenden über die Frage „Die Mitarbeiter haben Mitsprachrecht im Unternehmen, auch bei wichtigen Entscheidungen" abgefragt, da diese Formulierung die meisten Formen aktiver Partizipation einschließt. Da wir uns auf die initiale Nutzungsabsicht eines ECS vor dessen aktiver Implementierung beziehen, kann eine aktive Partizipation der Mitarbeitenden nicht direkt beobachtet werden. Erfragt wird hier also die Wirkung einer allgemeinen Partizipations- und Verfahrensgerechtigkeitserfahrung der einzelnen Mitarbeitenden innerhalb des Unternehmens. Hier können sich unterschiedliche positive und negative Erfahrungen des jeweiligen Befragten gegenseitig ausgleichen. Entsprechend können nur sehr starke oder kumulativ stark negative oder kumulativ stark positive Erfahrungen einer Person eine Wirkung auf dessen Nutzungsabsicht oder Widerstand erzielen.

\section{Methoden}

\subsection{Stichprobenbeschreibung}

Die Stichprobe besteht aus $N=212$ Teilnehmerinnen und Teilnehmern. Zum Zeitpunkt der Befragung waren 183 Befragte bei einem mittelständischen deutschen Fertigungsunternehmen angestellt, 29 bei einer mittelständischen deutschen IT-Firma. $62 \%$ waren männlich, $26 \%$ weiblich (alle Angaben waren freiwillig; $12 \%$ entschieden sich dazu, hier keine Angabe zu machen). Die Teilnehmer/innen kamen aus verschiedenen Bereichen der jeweiligen Firmen, wie beispielsweise Human Resources, Sales \& Services oder Supply Chain Management; das Bild der jeweiligen Zuständigkeiten ist sehr heterogen. Mit $33 \%$ besitzt der Großteil der Stichprobe einen Universitäts- oder Fachhochschulabschluss, $4 \%$ geben an, keinen Abschluss zu haben, und die restlichen Angaben bezogen sich auf eine abgeschlossene Berufsausbildung bzw. einen Fortbildungsabschluss (16\% keine Angabe). Durchschnittlich arbeiteten die Teilnehmenden 40,37 h in der Woche ( $\mathrm{SD}=7,86 ; \mathrm{Min}=3, \mathrm{Max}=70)$. Wichtig zu erwähnen ist es, dass sich die Teilnehmenden der beiden Unternehmen in einigen Aspekten signifikant voneinander unterscheiden (Geschlecht, Bildungsstand, wöchentliche Arbeitszeit).

\subsection{Vorgehen}

Die Befragung wurde im Herbst 2019 mittels eines OnlineFragebogens durchgeführt. Durch die Teilnahme der Unternehmen im Projektverbund wurde gemeinsam mit den Unternehmen eine Kommunikationsstrategie an die Mitarbeitenden entwickelt, und der Fragebogen wurde sowohl über das jeweilige Intranet als auch über die internen E-MailAdressen gestreut. Die Teilnahme am Fragebogen war freiwillig.

\subsection{Messinstrumente}

\subsubsection{Akzeptanz}

Widerstand gegenüber Veränderungen. Um Widerstand gegenüber Veränderungen zu messen, wurden acht Items der Resistance to Change-Skala von Oreg (2003) verwendet $(\alpha=0,74)$. Auf einer sechsstufigen Skala konnten die Teilnehmenden zwischen „stimme gar nicht $z u$ “ und „stimme stark zu “ auswählen. Ein Beispielitem ist: „Es stresst mich, wenn Dinge nicht nach Plan laufen. “

Nutzungsakzeptanz. Die Nutzungsakzeptanz wurde mit einem Item der Verhaltensabsicht (behavioral intention) nach Venkatesh et al. (2003) als Single-Item abgefragt. Das Item wurde wie folgt auf den ECS-Kontext angepasst: „Ich hätte vor, ECS zu nutzen." Zuvor wurde den Teilnehmenden erklärt, was sie sich unter ECS vorstellen können: „Stellen Sie sich vor, dass Ihr Unternehmen ein neues Programm für die digitale Zusammenarbeit einführt, nämlich ein Enterprise Collaboration System (ECS). Ein ECS ist ein Informationssystem, mit dem Mitarbeiter/innen und Teams Dokumente und Wissen einfacher miteinander teilen können. Das kann zum Beispiel eine interne OnlinePlattform sein, auf der Kollegen/innen gemeinsam an Dokumenten arbeiten, sich Nachrichten senden oder Termine koordinieren können."

\subsubsection{Stressoren}

Workload. Um Workload zu messen, wurde der Job Stress Questionnaire von Hamel und Bracken (1986) verwendet. Die fünf Items, welche auf einer siebenstufigen Likert-Skala zwischen „nie“ und ,immer" zu beantworten ist, zeigten 
als Skala eine interne Konsistenz von Cronbachs $\alpha=0,86$. Es sollten Fragen wie beispielsweise „, Wie oft bleibt Ihnen in Ihrem Job wenig Zeit, um alle Dinge zu erledigen?" eingeschätzt werden.

Rollenkonflikt. Für den Rollenkonflikt wurde ebenfalls der Job Stress Questionnaire herangezogen. Die zwei Items zeigten als Skala ein Cronbachs $\alpha$ von 0,76. „, Wie oft geben Personen, deren Aufforderungen Sie folgen sollten, Ihnen Aufgaben, die mit Ihren anderen Aufgaben im Konflikt stehen?" ist eines der Items.

Rollenambiguität. Ebenfalls im Job Stressor Questionnaire ist die Rollenambiguität. Hierzu wurden drei Items verwendet $(\alpha=0,56)$. Ein Beispielitem lautet ,Wie oft ist es Ihnen unklar, welche Verantwortlichkeiten Sie haben?".

Job-Komplexität. Als letzte Facette des Job Stress Questionnaires wurde die Job-Komplexität gemessen. Alle vier Items gemeinsam zeigten eine interne Konsistenz von Cronbachs $\alpha=0,57$. Ein Beispielitem hierzu ist „,Die Aufgaben in meiner Arbeit sind einfach und unkompliziert. “ (invertiert).

Emotionale Arbeit. Mit Hilfe der Skala von Brotheridge und Grandey (2002) wurde das Konstrukt der emotionalen Arbeit erfasst. Mit den sieben Items wurde gefragt, wie häufig bei der Arbeit eine Facette von emotionaler Arbeit abgeleistet werden muss, beispielsweise: „Müssen Sie bei Ihrer Arbeit ruhig bleiben, selbst wenn Sie innerlich aufgeregt sind?" $(\alpha=0,84)$. Die Frage wurde auf einer fünfstufigen Skala zwischen „nie“ und ,immer" beantwortet.

\subsubsection{Ressourcen}

Für die Erhebung der Ressourcen wurde der Work Design Questionnaire (Stegmann et al. 2010) verwendet. Im Folgenden werden die benutzten Unterskalen näher beschrieben. Alle Items der Unterskalen wurden auf einer sechsstufigen Skala zwischen „trifft gar nicht zu“ und „trifft genau $z u$ " eingeschätzt.

Rückmeldung durch die Tätigkeit. Mit drei Items wurde die Rückmeldung durch die Tätigkeit erhoben $(\alpha=0,72)$. Eines dieser Items lautet: „Bei der Ausführung meiner Arbeit kann ich leicht feststellen, wie gut ich arbeite. “

Rückmeldung durch andere. Die drei Items, welche Rückmeldung durch andere erhoben, hatten eine interne Konsistenz von Cronbachs $\alpha=0,84$. Ein Beispielitem lautet: „Andere Personen aus der Organisation geben mir Rückmeldung über die Effektivität meiner Arbeitsleistungen."

Wichtigkeit der Aufgabe. Die Wichtigkeit der Aufgabe wurde mittels vier Variablen erhoben $(\alpha=0,76)$. Unter anderem wurde beispielsweise folgendes Item aufgeführt: „Meine Arbeit ist bedeutsam und wichtig in einem größeren Zusammenhang. “

Ganzheitlichkeit der Aufgabe. Die interne Konsistenz der Skala für die Ganzheitlichkeit der Aufgabe lag bei Cron- bachs $\alpha=0,83$. Ein Beispielitem für die Skala ist: „Bei meiner Arbeit kann ich zu Ende bringen, was ich begonnen habe. “

Anforderungsvielfalt. Mittels vier Variablen wurde die Anforderungsvielfalt erhoben $(\alpha=0,85)$. Die Aussage „Bei dieser Arbeit setzt man viele verschiedene Kompetenzen ein. " bildet eines der vier Items.

Autonomie. Für die Subskalen Planung (,Ich kann meine Arbeit so planen, wie ich es möchte. “), Entscheidungen (, Meine Arbeit gewährt mir einen großen Entscheidungsspielraum.“) und Methode („Ich kann selbst entscheiden, mit welchen Mitteln ich ans Ziel komme. “) kamen jeweils vier Items zum Einsatz (Planung: $\alpha=0,83$, Entscheidungen: $\alpha=0,89$, Methode: $\alpha=0,84)$.

Soziale Unterstützung. In der Skala von Vinokur und Van Ryn (1993) werden mittels einer fünfstufigen Skala im Bereich „trifft nicht zu“ bis „trifft zu“ Einschätzungen über Aussagen wie „Meine Kolleginnen und Kollegen geben mir nützliche Informationen. “ abgegeben $(\alpha=0,89)$.

\subsubsection{Weitere Antezedenzien}

Partizipation. Das Item, das für die Erhebung der Partizipationsmöglichkeiten im Unternehmen genutzt wurde, stammt aus einer Untersuchung von Allen und Meyer (1990) und wurde für die vorliegende Studie angepasst. Den Teilnehmenden beurteilten die Aussage „Die Mitarbeiter haben Mitspracherecht im Unternehmen, auch bei wichtigen Entscheidungen. " auf einer siebenstufigen Skala.

Produktivität des gemeinsamen Arbeitens über Datenbanken. Unseres Wissens nach existiert keine evaluierte Skala zu Messung von Erfahrung mit Technologien. Zwar fragten beispielsweise Venkatesh und Davis (2000) Vorerfahrungen mit Technologien ab, jedoch bezog sich ihre Frage auf die Technik, deren Akzeptanz durch TAM2 ermittelt werden sollte. Da uns die Akzeptanz von ECS durch positive Erfahrungen mit Datenbanken, als Vorgängertechnik, interessiert, kann diese Skala nicht verwendet werden. Daher wurden positive Erfahrungen mit Datenbanken über das Item „Das gemeinsame Arbeiten an Dateien und Formularen (Texten, Tabellen, Prozessabläufen u.ä.) auf Datenplattformen halte ich für sehr produktiv " auf eigene Weise abgefragt, zuvor jedoch in einem kognitiven Pretest sowie im Rahmen eines Gesamt-Pretest des OnlineFragebogens geprüft.

\section{Ergebnisse}

\subsection{Auswertung}

Die statistische Auswertung erfolgte mit Hilfe des Programms IBM SPSS 25. Für die Auswertung der Hypothe- 
Tab. 2 Schrittweise Regression: Widerstand gegenüber Veränderungen x Job Stressoren (mit eingeschlossenen Variablen)

Table 2 Stepwise regression: resistance to change $\mathrm{x}$ job stressors (included variables)

\begin{tabular}{llllllll}
\hline Eingeschlossene Variablen & a & $B$ & $S E$ & $\beta$ & $t$ & $R^{2}$ & $\Delta R^{2}$ \\
\hline Modell 1 & - & - & - & - & 0,040 & - & - \\
(Konstante) & $2,364^{\mathrm{c}}$ & 0,278 & - & 8,514 & - & - & - \\
Job-Komplexität & $-0,146^{\mathrm{b}}$ & 0,063 & $-0,201$ & $-2,312$ & - & - \\
Modell 2 & - & - & - & - & 0,074 & 0,033 \\
(Konstante) & $2,011^{\mathrm{c}}$ & 0,320 & - & 6,284 & - & - & - \\
Job-Komplexität & $-0,135^{\mathrm{b}}$ & 0,062 & $-0,186$ & $-2,166$ & - & - & - \\
Rollenambiguität & $0,120^{\mathrm{b}}$ & 0,056 & 0,183 & 2,131 & - & - & - \\
\hline
\end{tabular}

$N=128$

$B$ unstandardisierter Regressionskoeffizient; $S E$ Standardfehler des unstandardisierten Regressionskoeffizienten; $\beta$ standardisierter

Regressionskoeffizient; $t$ ermittelter $t$-Wert; $R^{2}$ Anteil der aufgeklärten Varianz; $\Delta R^{2}$ Inkrementelle Änderung der aufgeklärten Varianz;

$\Delta F$ inkrementelle Änderung in $F$

${ }^{a}$ Jeder Schritt ist signifikant auf dem Niveau $p<0,05$

${ }^{\mathrm{b}} p<0,05$

${ }^{\mathrm{c}} p<0,001$

sen $1 \mathrm{a}, 1 \mathrm{~b}$ und $2 \mathrm{a}, 2 \mathrm{~b}$ wurden schrittweise hierarchische Regressionen mit paarweisem Ausschluss gewählt. Da die Hypothesen auf eine offen-explorative Weise viele Stressoren und Ressourcen als Prädiktoren miteinbeziehen, wurde die Schrittweise-Methode gewählt, um herauszufinden, welche Prädiktoren am meisten zur Varianzaufklärung beitragen.

Für die Hypothesen 3 und 4 wiederum wurden jeweils einfache lineare Regressionen gewählt, da es sich zusätzlich zum Work Design-Begriff um weitere wichtige Arbeits- und Organisationsmerkmale handelte, deren Einfluss separat geschätzt wurde. Die untersuchten Variablen folgen dabei näherungsweise einer Normalverteilung. Für mögliche Heteroskedastizität innerhalb der Modelle wurde mit heteroskedastie-robusten Standardfehlern gearbeitet. Aufgrund der geringen Fallzahlen wurden in der Auswertung auf sparsame Modelle (einzelne Regressionen je Ressourcen/Stressoren und Outcomes) zurückgegriffen.

\subsection{Kontrollvariablen}

Mit Hilfe von MANOVAs wurde getestet, ob Alter und Geschlecht einen signifikanten Einfluss auf alle beteiligten Variablen hatten (Alter und Geschlecht als unabhängige Variablen, Prädiktoren und Outcome-Variablen als abhän- gige Variablen). Dies bestätigte sich nicht. Somit wurden die Regressionen zur Hypothesentestung ohne Alter und Geschlecht als Kontrollvariablen berechnet. Da die Stichprobe insgesamt hochgebildet ist, wurde Bildung nicht als Kontrollvariable hinzugezogen, um dadurch die Ergebnisse nicht zu verzerren.

\subsection{Hypothesentestung}

In der schrittweisen Regression der Job-Stressoren mit Widerstand gegenüber Veränderungen zeigten Job-Komplexität und Rollenambiguität signifikante Zusammenhänge mit Widerstand. Dementsprechend wird Hypothese 1a teilweise bestätigt. Der Zusammenhang zwischen Job-Komplexität und Widerstand ist negativ, da die Skala invertiert ist; dementsprechend zeigt der Zusammenhang in die erwartete Richtung (Personen mit hohem Widerstand gegenüber Veränderungen berichten auch von hoher Job-Komplexität). Die genauen Werte sind Tab. 2 zu entnehmen.

In der Regression der Job-Ressourcen auf Widerstand gegenüber Veränderungen wird der Zusammenhang zwischen der Entscheidung-Subskala der Autonomie und dem Widerstand signifikant. Der negative Zusammenhang geht ebenfalls in eine zu erwartende Richtung (Personen, wel-

Tab. 3 Schrittweise Regression: Widerstand gegenüber Veränderungen x Job Ressourcen (mit eingeschlossenen Variablen)

Table 3 Stepwise regression: resistance to change $\mathrm{x}$ job resources (included variables)

\begin{tabular}{llllllll}
\hline Eingeschlossene Variablen $^{\mathrm{a}}$ & $B$ & $S E$ & $\beta$ & $t$ & $R^{2}$ & $\Delta R^{2}$ & $\Delta F$ \\
\hline- & - & - & - & - & 0,160 & - & 26,381 \\
(Konstante) & $2,619^{\mathrm{b}}$ & 0,179 & - & 14,665 & - & - & - \\
Autonomie Entscheidungen & $-0,254^{\mathrm{b}}$ & 0,049 & $-0,399$ & $-5,136$ & - & - & - \\
\hline
\end{tabular}

$N=140$

$B$ unstandardisierter Regressionskoeffizient; SE Standardfehler des unstandardisierten Regressionskoeffizienten; $\beta$ standardisierter

Regressionskoeffizient; $t$ ermittelter $t$-Wert; $R^{2}$ Anteil der aufgeklärten Varianz; $\Delta R^{2}$ Inkrementelle Änderung der aufgeklärten Varianz;

$\Delta F$ inkrementelle Änderung in $F$

a Jeder Schritt ist signifikant auf dem Niveau $p<0,05$

${ }^{\mathrm{b}} p<0,001$. 
che einen hohen Widerstand gegenüber Veränderungen zeigen, berichteten weniger, dass sie Entscheidungsautonomie besitzen). Dementsprechend steht eine Job-Ressource, die Entscheidungsfacette der Autonomie, im Zusammenhang mit Widerstand gegenüber Veränderungen, und somit wird Hypothese $1 b$ nicht abgelehnt. Die genauen Werte sind Tab. 3 zu entnehmen.

Wir konnten keine Zusammenhänge zwischen Job-Stressoren und der Verhaltensabsicht, ECS zu nutzen, finden. Dementsprechend muss Hypothese 2a verworfen werden.

In der Regression der Job-Ressourcen auf die Verhaltensabsicht, ECS zu nutzen, wurden die Zusammenhänge zwischen der Wichtigkeit der Tätigkeit und der Verhaltensabsicht sowie zwischen der Rückmeldung durch die Tätigkeit und der Verhaltensabsicht signifikant. Hier ist interessant zu beobachten, dass der Zusammenhang von Wichtigkeit der Tätigkeit und der Verhaltensabsicht, ECS zu nutzen, positiv ist. Das würde bedeuten, dass Personen, die angeben, das ECS nutzen zu wollen, ihre Tätigkeit als bedeutungsvoll empfinden. Dahingegen ist der gefundene Zusammenhang zwischen Rückmeldung aus der Tätigkeit und Verhaltensabsicht negativ; also empfinden Personen, die angeben, das ECS nutzen zu wollen, ihre Tätigkeiten tendenziell weniger so, als ob die Ausführung der Tätigkeit selbst Auskunft über die Qualität der ausgeführten Arbeitsaufgaben gibt. Es gibt also, auch wenn auf unterschiedliche Arten, einen Zusammenhang zwischen der ressourcenreichen Gestaltung der Arbeit und der Verhaltensintention, das ECS zu nutzen. Dementsprechend wird Hypothese $2 \mathrm{~b}$ nicht abgelehnt. Die genauen Werte sind in Tab. 4 dargestellt.

Der gefundene positive Zusammenhang zwischen der wahrgenommenen Produktivität der Arbeit über Datenbanken und der Nutzungsabsicht für ECS (siehe Tab. 5) deutet darauf hin, dass positive Vorerfahrungen mit Techniken begünstigend auf die Adaption der neuen Technologie wirken, womit unsere Hypothese 3 nicht verworfen werden kann.

Entgegen unserer Erwartung zeigt die Partizipation der Mitarbeitenden keine statistisch signifikanten Einflüsse auf die Nutzungsabsicht von ECS durch die Mitarbeitenden. Auch konnte kein Zusammenhang zwischen Partizipation und Widerstand gegenüber Veränderungen gefunden werden. Somit muss Hypothese 4 als Ganzes verworfen werden.

Tab. 4 Schrittweise Regression: Verhaltensintention x Job-Ressourcen (nur eingeschlossene Variablen)

Table 4 Stepwise regression: behavioral intention $\mathrm{x}$ job stressors (included variables)

\begin{tabular}{llllllll}
\hline Eingeschlossene Variablen & a & $B$ & $S E$ & $\beta$ & $t$ & $R^{2}$ & $\Delta R^{2}$ \\
\hline Modell 1 & - & - & - & - & 0,036 & - & - \\
(Konstante) & $2,891^{\mathrm{c}}$ & 0,173 & - & 16,755 & - & - & - \\
Wichtigkeit der Tätigkeit & $0,120^{\mathrm{b}}$ & 0,053 & 0,190 & 2,287 & - & - \\
Modell 2 & - & - & - & - & 0,064 & 0,027 \\
(Konstante) & $3,126^{\mathrm{c}}$ & 0,207 & - & 15,116 & - & - & - \\
Wichtigkeit der Tätigkeit & $0,158^{\mathrm{b}}$ & 0,055 & 0,250 & 2,856 & - & - & - \\
Rückmeldung durch die Tätigkeit & $-0,118^{\mathrm{b}}$ & 0,059 & $-0,176$ & $-2,011$ & - & - & - \\
\hline
\end{tabular}

$N=140$

$B$ unstandardisierter Regressionskoeffizient; SE Standardfehler des unstandardisierten Regressionskoeffizienten; $\beta$ standardisierter

Regressionskoeffizient; $t$ ermittelter $t$-Wert; $R^{2}$ Anteil der aufgeklärten Varianz; $\Delta R^{2}$ Inkrementelle Änderung der aufgeklärten Varianz;

$\Delta F$ inkrementelle Änderung in $F$

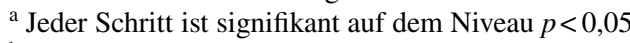

b $p<0,05$

${ }^{\mathrm{c}} p<0,001$

Tab. 5 Lineare Regression: Verhaltensabsicht x Produktivität über Datenbanken

Table 5 Linear regression: behavioral intention $\mathrm{x}$ productivity with databases

\begin{tabular}{lllllll}
\hline Prädiktoren & $B$ & $S E$ & $\beta$ & $t$ & $R^{2}$ & $F$ \\
\hline- & - & - & - & - & 0,078 & 15,552 \\
(Konstante) & $2,479^{\mathrm{b}}$ & 0,205 & - & 12,113 & - & - \\
Produktivität über Datenbanken & $0,171^{\mathrm{b}}$ & 0,043 & 0,279 & 3,944 & - & - \\
\hline
\end{tabular}

$N=185$

$B$ unstandardisierter Regressionskoeffizient; SE Standardfehler des unstandardisierten Regressionskoeffizienten; $\beta$ standardisierter

Regressionskoeffizient; $t$ ermittelter $t$-Wert; $R^{2}$ Anteil der aufgeklärten Varianz; $\Delta R^{2}$ Inkrementelle Änderung der aufgeklärten Varianz;

$\Delta F$ inkrementelle Änderung in $F$

${ }^{a}$ Jeder Schritt ist signifikant auf dem Niveau $p<0,05$

${ }^{\mathrm{b}} p<0,001$ 


\section{Diskussion}

\subsection{Zusammenfassung und Interpretation der Ergebnisse}

In den Hypothesen 1 und 2, die nach Jobcharakteristika im Rahmen der Work Design-Theorie suchten, welche einen Zusammenhang mit Widerstand gegenüber Veränderungen und der Absicht, das neue ECS zu nutzen, aufweisen, konnten folgende Zusammenhänge gefunden werden: Die Stressoren Job-Komplexität und Rollenambiguität hängen mit Widerstand gegenüber Veränderungen zusammen. Der Zusammenhang zwischen der Komplexität und dem Widerstand gegenüber Veränderungen ist negativ, da die Skala invertiert ist, und der der Zusammenhang zwischen Rollenambiguität und Widerstand ist positiv. Auch die Ressource Autonomie über Entscheidungsprozesse hängt mit Widerstand gegenüber Veränderung zusammen, diesmal negativ. Somit werden die Hypothesen $1 \mathrm{a}$ und $1 \mathrm{~b}$ nicht verworfen. Die Richtungen der Zusammenhänge lassen sich mit dem JDRM vergleichen; anstatt der Stressoutcomes wurden hier Zusammenhänge mit einem Widerstandkonstrukt gefunden. Generell stellt sich für die weitere Forschung die Frage, inwieweit hier Effekte und Wirkmechanismen über Affektund Befindenskonstrukte aufgeklärt werden können, da es bereits mehrere Befunde gibt, in denen sich Arbeitscharakteristika, Befindensoutcomes wie Arbeitszufriedenheit und die Einführung von neuen Technologien gegenseitig beeinflussen (Schraeder et al. 2006; Morris und Venkatesh 2010). Dass die Entscheidungsfacette von Autonomie mit Widerstand gegenüber Veränderungen zusammenhängt, kann als valide betrachtet werden: Personen, die Veränderungen kritisch gegenüberstehen, könnten sich beispielsweise bewusst Arbeitsaufgaben und -plätze suchen, an denen sie die Freiheit haben, selbst zu entscheiden, ob sich etwas verändern soll. Darüber hinaus werden bereits im Bewerbungsprozess Personen mit einer kritischen Einstellung gegenüber Veränderungen aussortiert, wenn diese Mentalität nicht zur vakanten Stelle oder der Betriebsmentalität passt. Interessant zu sehen ist auch, dass von Stressorenseite mit der Rollenambiguität sowohl eine Facette der Rollentheorie sowie ein traditioneller Arbeitsstressor wie die Komplexität Zusammenhänge mit Widerstand gegenüber Veränderungen aufweisen. Hier ist zu untersuchen, warum soziale Stressoren wie emotionale Arbeit keinen Zusammenhang aufweisen und ob man vielleicht einen anderen sozialen Stressor zur Rate ziehen sollte.

In den Hypothesen $2 \mathrm{a}$ und $2 \mathrm{~b}$ wurde untersucht, inwieweit Stressoren und Ressourcen mit der Absicht, das ECS zu nutzen, in Zusammenhang stehen. Hier wurde kein $\mathrm{Zu}$ sammenhang mit einem Stressor signifikant, sodass die Hypothese $2 \mathrm{a}$ verworfen wurde. Hypothese $2 \mathrm{~b}$ wurde nicht verworfen, da die Ressourcen Wichtigkeit der Tätigkeit so- wie Rückmeldung durch die Tätigkeit einen signifikanten Zusammenhang zur Verhaltensabsicht, das ECS zu nutzen, aufweisen. Dies spricht dafür, dass die konkrete Ausgestaltung der Tätigkeit, zu dessen Bearbeitung das ECS genutzt werden soll, eine hohe Bedeutung für die Nutzungsabsicht des ECS hat. Die Tätigkeit sollte sowohl für den individuellen Mitarbeitenden eine hohe Relevanz aufzeigen als auch klar genug sein, um Rückmeldung von der Ausführung der Arbeit selbst zu erhalten. Dies könnte beispielsweise der Fall sein, da die Kommunikation über spezifische Arbeitsschritte einfacher ist und Rückmeldungen leicht über ECS vermittelt werden können. Überraschend ist, dass keine Beziehungsfacette von Work Design wie soziale Unterstützung oder Rückmeldung durch andere signifikante Zusammenhänge zeigte. Das spricht dafür, dass man die sozialen Aspekte der Arbeit immer noch eher face-to-face ausleben möchte und die Akzeptanz, hierfür ECS zu nutzen, eher niedriger ist. ECS könnte von den Mitarbeitenden auch als reines Arbeitsmittel begriffen werden, welches nur für die Kommunikation über formelle Arbeitsinhalte genutzt wird, die nicht relationaler Natur sind.

Bei der Prüfung der Hypothese 3 zeigte sich ein signifikanter Zusammenhang zwischen der Nutzungsabsicht des ECS und den Vorerfahrungen mit Technologien, womit die Hypothese nicht verworfen werden kann. Offen bleibt die Frage, ob Personen, welche ohnehin bereits eine höhere Technikaffinität aufweisen stärker auf Datenbanken zurückgreifen. Da Datenbanken jedoch eine in deutschen Betrieben fest integrierte Technik darstellen gehen wir davon aus, dass die Nutzung für die Angestellten nicht optional ist, sondern dass sich viele Geschäftsprozesse ohne diese Technik nicht mehr erledigen lassen.

Die Hypothese 4 musste verworfen werden, da keine $\mathrm{Zu}-$ sammenhänge zwischen Partizipation und der Nutzungsabsicht von ECS bzw. dem Widerstand gegenüber Veränderungen ermittelt wurden. Wir vermuten, dass die Partizipation der Mitarbeitenden im Einführungsprozess einen stark an den tatsächlichen Prozess einer wichtigen Veränderung am Arbeitsplatz gebundenen Faktor darstellt. In einer Abfrage einer Nutzungsabsicht, hier von ECS, scheint dieser nicht bedacht zu werden oder keinen Einfluss auszuüben. Es müsste eine sehr umfängliche Partizipation oder starke und wiederholte Verletzungen von Partizipationsansprüchen bestehen, um auf eine unspezifisch abgefragte Nutzungsabsicht von Technik Wirkungen zu erzielen. Dies ist bei den Befragten nicht der Fall. Es ist aber auch nicht ausgeschlossen, dass Partizipation bei der Messung einer tatsächlichen Einführung eines ECS ein Effekt beizumessen ist.

\subsection{Limitationen}

Die vorliegende Studie unterliegt einigen methodischen Limitationen. Vor allem zu erwähnen ist, dass es sich bei 
den Ergebnissen um Zusammenhänge zwischen Variablen aus derselben Querschnittuntersuchung mit Selbstreport handelt. Hier kann ein Common Method Bias nicht ausgeschlossen werden (Podsakoff et al. 2003;), der die Zusammenhänge möglicherweise künstlich aufgebläht hat. Auch war die interne Konsistenz der Messinstrumente nicht ganz einwandfrei; die Skala für Job-Komplexität zeigte ein niedriges Cronbachs Alpha von 0,64, was bedeutet, dass die Items der Skala möglicherweise nicht reliabel dasselbe Konstrukt messen. Hierbei ist Vorsicht bei der Interpretation der Ergebnisse geboten. Da es sich um Regressionen aus einem Messzeitpunkt handelt, muss erwähnt werden, dass aus den Zusammenhängen keine Kausalitäten abgeleitet werden können. Die Richtung der gefundenen Zusammenhänge sollte mit einem weiteren Messzeitpunkt untersucht werden. Außerdem ist Widerstand gegenüber Veränderungen eine Trait-Variable, die nicht als ein klassisches Outcome gesehen werden kann, da es sich um eine dispositionelle Einstellung eines Individuums handelt, die nicht nur auf den Arbeitskontext zugeschnitten sein muss. Durch ein Studiendesign, welches eine Kausalitätsprüfung zulässt, sollte überprüft werden, ob Widerstand eher eine Prädiktor- oder eine Outcomevariable darstellt.

Die Auswertung der Ergebnisse fand pro Variable mit variierender Teilnehmendenanzahl statt. Diese Auswertungsart (Schrittweise-Regressionen, paarweiser Ausschluss) wurde gewählt, um so viel Varianz wie möglich und damit auch so viele Einflussvariablen auf die Technikakzeptanz wie möglich zu identifizieren. Dies wurde getan, um möglichst viele Ansätze für die weitere Forschung zu kreieren. Jedoch sind die Ergebnisse etwas eingeschränkter vergleichbar, da ein Raum für Signifikanzen von Scheinzusammenhängen eröffnet wurde. Beispielsweise ist der Koeffizient der Regression von Rollenambiguität auf Widerstand gegenüber Veränderungen mit $\mathrm{B}=0,12(p<0,05)$ gering; falls es hier zu einer zufälligen Signifikanz kam, kann man nicht davon ausgehen, dass es bei hoher Rollenambiguität auch zu hohem Widerstand kommt. Die Zusammenhänge sollten demnach in Replikationen und weiterer Forschung gesichert werden. Auch wurde hier mit der Nutzungsabsicht eines ECS im Speziellen gearbeitet. Die Generalisierung der Nutzungsakzeptanz dieser spezifischen Technologie auf eine allgemeine Techniknutzungsakzeptanz sollte nur vorsichtig erfolgen. Der spezifische Anwendungsfall zeigt hier nur Ansatzpunkte auf und sollte mit anderen Technologien repliziert werden.

\subsection{Implikationen für Forschung und Praxis}

Die vorliegende Studie gibt erste Hinweise darauf, dass Work Design und die Erfahrungen von Nutzerinnen und Nutzern, welche von Organisationen beeinflusst werden können, mit der Akzeptanz von Technik zusammenhängen (in der vorliegenden Studie anhand vom Anwendungsfall der ECS-Einführung untersucht). Durch die Zusammenhänge zwischen Work Design-Komponenten und Komponenten der Technikakzeptanz stellt sich heraus, dass bei der Einführung neuer ECS, oder generalisiert Kommunikationstechnologien im Allgemeinen, die Arbeitsgestaltung an sich mitgedacht werden muss. Unter welchen Bedingungen oder in welchen Zeiträumen des Einführungsprozesses ist noch zu erforschen. Jedoch bieten diese Ergebnisse in diesem speziellen Kontext dieser Anknüpfungspunkte, hier näher einzugehen. In Organisationen selbst sollte bei der Einführung dementsprechend nicht nur der Umgang mit der Technologie selbst vermittelt werden, sondern auch (beispielsweise durch Kurse oder Workshops) die Arbeitsgestaltung mit und um die Technologie behandelt werden. Im Einführungsprozess sind ebenfalls die Vorerfahrungen der Mitarbeitenden zu bedenken. Bei der Implementierung von I+K Techniken in eine nicht IT-affine Arbeitsumgebung ist tendenziell mit mehr Vorbehalten zu rechnen, welche frühzeitig, etwa durch Kurse oder schrittweise Einführung von Vorgängertechniken entgegengewirkt werden sollte. Dies ist eine wichtige Erkenntnis, wenn es darum geht, neue Technologien wie ECS in Organisationen einzuführen. Wenn Organisationen und Unternehmen wollen, dass die neue Technologie gewinnbringend eingesetzt werden soll, scheint es wichtig zu sein, sich zuvor Gedanken um die Gestaltung der Arbeit und die Erfahrungen der Mitarbeitenden am Arbeitsplatz zu machen. Die Initiative „SMART Work Design“ des Centre für Transformative Work Design (https://www.smartworkdesign.com.au/) hilft Unternehmen mit Praxisbeispielen und Erklärungen, wie man Work Design-Prinzipien basierend auf der Work Design-Forschung von Parker und Kolleg/innen (Parker 2014; Parker et al., 2017) besser umsetzen kann. Nicht nur für die Verbesserung des Befindens von Mitarbeitenden und damit zur Steigerung von Leistung sowie zur Senkung von Arbeitsrisiken und Fluktuation, sondern auch für die Steigerung von Technikakzeptanz kann Work Design ein wichtiger Bestandteil sein.

Um hierzu jedoch genau festzustellen, ob solche Work Design-Elemente und die aufzubauenden Erfahrungen der Grund für die positive Einstellung zur neuen Technologie sind, sollte weiterhin die Kausalität der Zusammenhänge überprüft werden. Hierbei sollte bei einer zweiten Befragung untersucht werden, inwiefern die echte Nutzung des neuen ECS ausfällt. Spannend hierbei zu beobachten und dringend zu kontrollieren ist der äußere Zwang zur Nutzung solcher Technologien im Projektkontext, der aus der Pandemie und damit einhergehend ggf. der Arbeit aus dem Home-Office entspringt.

Wie auch in der Ergebnisdiskussion erwähnt sollte in weiteren Studien die Rolle von Stress, Motivation, Gesundheit, Arbeitszufriedenheit und Engagement als Befindens- 
variablen auf der einen Seite, sowie positiver und negativer Affekt auf der anderen Seite berücksichtigt werden. Da sich die klassischen Work Design-Ansätze auf motivationale und Befindensvariablen beziehen, ist es denkbar, dass diese eine größere Rolle im Zusammenhang zwischen Work DesignElementen und der Technikakzeptanz haben.

\subsection{Fazit}

Die vorliegende Studie untersucht die Bedeutung von Work Design, Partizipation im Unternehmen und Technik-Vorerfahrungen für Technikakzeptanz. Diese Einflussfaktoren wurden gewählt, da sie von Organisationen beeinflussbar sind, sodass praktische Implikationen abgeleitet werden können. Die Ergebnisse der Studie liefern erste Hinweise darauf, dass es Zusammenhänge zwischen Facetten von Work Design bzw. Vorerfahrungen mit Technik und der Nutzungsakzeptanz gibt. Diese Zusammenhänge zeigen, dass Work Design und die Erfahrungen der Mitarbeitenden bei der Einführung neuer Technologie berücksichtigt werden sollten. In weiteren Forschungsarbeiten sollte die Kausalität dieser Zusammenhänge untersucht werden, und die Zusammenhänge bei der Einführung anderer Technologien repliziert werden. Auch individuelle Befindensoutcomes könnten bei den Wirkmechanismen eine Rolle spielen.

Förderung Die vorliegende Veröffentlichung entstand im Rahmen des Projekts „Digitale Kooperationssysteme im Mittelstand: Innovative Kommunikations- und Kooperationsprozesse in der digitalen Arbeitswelt (KoMiK)“. Dieses Forschungs- und Entwicklungsprojekt wird im Rahmen des Programms ,Zukunft der Arbeit“ vom Bundesministerium für Bildung und Forschung (BMBF) und dem Europäischen Sozialfonds (ESF) gefördert und vom Projektträger Karlsruhe (PTKA) betreut. Die Verantwortung für den Inhalt dieser Veröffentlichung liegt bei den Autorinnen und Autoren.

Funding Open Access funding enabled and organized by Projekt DEAL.

Open Access Dieser Artikel wird unter der Creative Commons Namensnennung 4.0 International Lizenz veröffentlicht, welche die Nutzung, Vervielfältigung, Bearbeitung, Verbreitung und Wiedergabe in jeglichem Medium und Format erlaubt, sofern Sie den/die ursprünglichen Autor(en) und die Quelle ordnungsgemäß nennen, einen Link zur Creative Commons Lizenz beifügen und angeben, ob Änderungen vorgenommen wurden.

Die in diesem Artikel enthaltenen Bilder und sonstiges Drittmaterial unterliegen ebenfalls der genannten Creative Commons Lizenz, sofern sich aus der Abbildungslegende nichts anderes ergibt. Sofern das betreffende Material nicht unter der genannten Creative Commons Lizenz steht und die betreffende Handlung nicht nach gesetzlichen Vorschriften erlaubt ist, ist für die oben aufgeführten Weiterverwendungen des Materials die Einwilligung des jeweiligen Rechteinhabers einzuholen.

Weitere Details zur Lizenz entnehmen Sie bitte der Lizenzinformation auf http://creativecommons.org/licenses/by/4.0/deed.de.

\section{Literatur}

Allen NJ, Meyer JP (1990) The measurement and antecedents of affective, continuance and normative commitment to the organization. J Occup Psychol 63(1):1-18. https://doi.org/10.1111/j.20448325.1990.tb00506.x

Bakker AB, Demerouti E (2007) The job demands-resources model: state of the art. Journal of Managerial Psych 22(3):309-328. https://doi.org/10.1108/02683940710733115

Bakker AB, Demerouti E (2017) Job demands-resources theory: Taking stock and looking forward. J Occup Health Psychol 22(3):273-285. https://doi.org/10.1037/ocp0000056

Battistelli A, Montani F, Odoardi C (2013) The impact of feedback from job and task autonomy in the relationship between dispositional resistance to change and innovative work behaviour. Eur J Work Organ Psychol 22(1):26-41. https://doi.org/10.1080/ 1359432X.2011.616653

Brotheridge CM, Grandey AA (2002) Emotional labor and burnout: comparing two perspectives of ,people work“. J Vocat Behav 60(1):17-39

Cooper R, Zmud R (1990) Information technology implementation research: a Technolgical diffusion Apporach. Manag Sci 36(2):123-139

Compeau DR, Higgins CA (1995) Computer Self-Efficacy: Development of a Measure and Initial Test. MIS Quarterly 19(2):189-211

Demerouti E, Bakker AB, Nachreiner F, Schaufeli WB (2001) The job demands-resources model of burnout. J Appl Psychol 86(3):499-512. https://doi.org/10.1037/0021-9010.86.3.499

Hackman JR (1980) Work redesign and motivation. Prof Psychol 11(3):445-455. https://doi.org/10.1037/0735-7028.11.3.445

Hackman JR, Lawler EE (1971) Employee reactions to job characteristics. J Appl Psychol 55(3):259-286. https://doi.org/10.1037/ h0031152

Hackman JR, Oldham GR (1975) Development of the job diagnostic survey. J Appl Psychol 60(2):159-170. https://doi.org/10.1037/ h0076546

Hackman JR, Oldham GR (1976) Motivation through the design of work: test of a theory. Organ Behav Hum Perform 16(2):250-279. https://doi.org/10.1016/0030-5073(76)90016-7

Hamel K, Bracken D (1986) Factor structure of the Job Stress Questionnaire (JSQ) in three occupational groups. Educ Psychol Meas 46(3):777-786. https://doi.org/10.1177/0013164486463036

Heidenreich S (2012) Innovations-doomed to fail?: Investigating passive and active innovation resistance. Kovač,

Jeung DY, Lee HO, Chung WG, Yoon JH, Koh SB, Back CY, Chang SJ (2017) Association of emotional labor, self-efficacy, and type A personality with burnout in Korean dental hygienists. J Korean Med Sci 32(9):1423. https://doi.org/10.3346/jkms.2017.32.9. 1423

Johnson JV, Hall EM (1988) Job strain, work place social support, and cardiovascular disease: a cross-sectional study of a random sample of the Swedish working population. Am J Public Health 78(10):1336-1342

Karasek RA (1979) Job Demands, Job decision latitude, and mental strain: Implications for job redesign. Adm Sci Q 24(2):285. https://doi.org/10.2307/2392498

Karasek RA (1998) Demand/Control Model: a social, emotional, and physiological approach to stress risk and active behaviour development. In: Stellman JM (Hrsg) Encyclopedia of occupational health and safety. ILO, Geneva, S 34.06-34.14

Katsikea E, Theodosiou M, Perdikis N, Kehagias J (2011) The effects of organizational structure and job characteristics on export sales managers' job satisfaction and organizational commitment. J World Bus 46(2):221-233. https://doi.org/10.1016/j.jwb.2010. 11.003 
Koch M, Richter A (2009) Enterprise 2.0: Planung, Einführung und erfolgreicher Einsatz von Social Software in Unternehmen. Oldenbourg, München

Kollmann T (1999) Akzeptanzprobleme neuer Technologien-Die Notwendigkeit eines dynamischen Untersuchungsansatzes. In: Electronic Commerce. Gabler, Wiesbaden. S 27-45

Lauer T (2010) Change management. Springer, Berlin Heidelberg

Lee D, Rhee Y, Dunham RB (2009) The role of organizational and individual characteristics in technology acceptance. Int $\mathrm{J}$ Hum Comput Interact 25(7):623-646. https://doi.org/10.1080/ 10447310902963969

Leventhal G (1980) What should be done with equity theory? New approaches to the study of fairness in social relationships. In: Gergen K, Greenberg M, Willis R (Hrsg) Social exchange. Advances in theory and research. Plenum, New York, S 27-55

Levinson H, Kahn RL, Wolfe DM, Quinn RP, Snoek JD, Rosenthal RA (1965) Organizational stress: studies in role conflict and ambiguity. Adm Sci Q 10(1):125. https://doi.org/10.2307/2391654

Mérida-López S, Extremera N, Rey L (2017) Emotion-regulation ability, role stress and teachers' mental health. Occupat Med 67(7):540-545. https://doi.org/10.1093/occmed/kqx125

Morris MG, Venkatesh V (2010) Job characteristics and job satisfaction: understanding the role of enterprise resource planning system implementation. MISQ 34(1):143. https://doi.org/10.2307/ 20721418

Olivares-Faúndez VE, Gil-Monte PR, Mena L, Jélvez-Wilke C, Figueiredo-Ferraz H (2014) Relationships between burnout and role ambiguity, role conflict and employee absenteeism among health workers. Terapia Psicológica 32(2):111-120. https://doi.org/10. 4067/S0718-48082014000200004

Oreg S (2003) Resistance to change: developing an individual differences measure. J Appl Psychol 88(4):680-693. https://doi.org/ 10.1037/0021-9010.88.4.680

Parker SK (2014) Beyond motivation: job and work design for development, health, ambidexterity, and more. Annu Rev Psychol 65(1):661-691. https://doi.org/10.1146/annurev-psych-010213115208

Parker SK, Morgeson FP, Johns G (2017) One hundred years of work design research: looking back and looking forward. J Appl Psychol 102(3):403-420. https://doi.org/10.1037/ap10000106

Podsakoff PM, MacKenzie SB, Lee J-Y, Podsakoff NP (2003) Common method biases in behavioral research: a critical review of the literature and recommended remedies. J Appl Psychol 88(5):879-903. https://doi.org/10.1037/0021-9010.88.5.879
Schraeder M, Swamidass PM, Morrison R (2006) Employee involvement, attitudes and reactions to technology changes. J Leadersh Organ Stud 12(3):85-100. https://doi.org/10.1177/10717 9190601200306

Schreder G, Smuc M, Siebenhandl K, Mayr E (2013) Age and computer self-efficacy in the use of digital technologies: an investigation of prototypes for public self-service terminals. In: Antona M, Stephanidis C (Hrsg) Universal access in human-computer interaction. Methods, technologies, and users 7th International Conference, UAHCI 2013, Held as Part of HCI International 2013, Las Vegas, July 21-26, 2013. Lecture Notes in Computer Science, vol. 10907, vol. 8010. Springer, Cham, S 221-230 (Proceedings, Part II)

Schubert P, Williams S (2013) The concept of social business: oxymoron or sign of a changing work culture? 26th Bled eConference. eInnovations: Challenges and Impacts for Individuals, Organizations and Society, June 9-June 13, 2013 Slovenia, Bled

Schwab RL, Iwanicki EF (1982) Perceived role conflict, role ambiguity, and teacher burnout. Educ Adm Q 18(1):60-74

Stegmann S, van Dick R, Ullrich J, Charalambous J, Menzel B, Egold N, Wu TT-C (2010) Der work design questionnaire. Z Arbeits Organisationspsychol 54(1):1-28. https://doi.org/10.1026/ 0932-4089/a000002

Venkatesh V, Bala H (2008) Technology acceptance model 3 and a research agenda on interventions. Decis Sci 39(2):273-314

Venkatesh V, Davis FD (2000) A theoretical extension of the technology acceptance model: four longitudinal field studies. Manage Sci 46(2): 186-204

Venkatesh V, Morris MG, Davis GB, Davis FD (2003) User acceptance of information technology: toward a unified view. MIS Quarterly 27(3):425-478. https://doi.org/10.2307/30036540

Vinokur AD, Van Ryn M (1993) Social support and undermining in close relationships: their independent effects on the mental health of unemployed persons. J Pers Soc Psychol 65(2):350-359. https://doi.org/10.1037/0022-3514.65.2.350

Wong A, Ng P, Fung Lo M (2018) Factors that affect the acceptance of new technologies in the workplace: a cross case analysis between two universities. Int J Educ Dev Using Inf Commun Technol 14(3):209-220

Zapf D (2002) Emotion work and psychological well-being: a review of the literature and some conceptual considerations. Hum Resour Manag Rev 12(2):237-268. https://doi.org/10.1016/S10534822(02)00048-7 